\title{
On the fast movements of the thermosphere: an interpretation
}

\author{
J. Lilensten ${ }^{1}$, P. O. Amblard ${ }^{2}$ \\ ${ }^{1}$ Laboratoire de Planétologie de Grenoble; Observatoire des Sciences de l'Univers de Grenoble; Bâtiment D de Physique; \\ BP 53; 38041 Grenoble cedex 9. France \\ ${ }^{2}$ LIS-INPG, BP 46, 38402 Saint Martin d'Hères cedex, France
}

Received: 28 June 2000 / Revised: 27 September 2000 / Accepted: 4 October 2000

\begin{abstract}
We examine the oscillations of the meridional neutral wind in the $\mathrm{F}$ region as seen by the EISCAT radar. We propose an interpretation in term of eddies (tourbillons) of typical size of a few tens to a few hundreds of kilometers. The observed rotation velocity is a few hundreds of meters per second. We suggest that the tourbillons are a common feature of thermospheric movements. We propose an optical experiment to check the validity of this assumption.
\end{abstract}

Key words: Atmospheric composition and structure (thermosphere - composition and chemistry) Ionosphere (ionosphere - atmosphere interactions)

\section{Introduction}

This work started with the coordinated measurements of two instruments, an interferometer and a geophysical radar.

The interferometer is a French instrument named MICADO. It was devoted to the study of the highaltitude atmosphere through observation of two different emission lines: O1D at $630 \mathrm{~nm}$ and O1S at $557.7 \mathrm{~nm}$ (Thuiller and Hersé, 1988). In this instrument, monochromatic light generates an interferogram by changing the interferometer optical path difference by several wavelengths. Atoms and molecules are put into metastable states by different excitation processes such as collisions and recombination. These atoms and molecules emit light and are also transported according to the various movements of the neutral or ionized species. These movements induce a Doppler effect measured from a phase change between an interferogram corresponding to a zero wind condition and another one for a given direction of measurement. From this instrument,

Correspondence to: J. Lilensten

e-mail: jean.lilensten@planeto.obs.ujf.grenoble.fr the neutral winds, i.e., zonal, meridional and vertical could be deduced. It was located in Sodankylä (Finland) during the winters of 1989 and 1990, and in Tromsø (Norway) in 1991.

The geophysical radar is EISCAT. The EISCAT scientific association is an international research organization operating three geophysical research Incoherent Scatter Radar systems, an Ionospheric Heater and a Dynasonde in Northern Scandinavia.

Two of the radar transmitters are located close to the city of Tromsø in Norway, while the third, the EISCAT Svalbard Radar (ESR) is located near Longyearbyen. We focus on data from the auroral region radar over Tromsø. One can find most of the useful explanations on the diverse modes of the EISCAT radar in Rishbeth and Van Eycken (1993). The analysis of the scattered signal allows us to deduce the ion and electron temperatures, the ion velocity parallel to the magnetic field line, and the electron density. The ion velocity does not depend on the shape of the spectrum, but on its Doppler decay. The total power of the spectrum gives an estimate of the electron density. Its width leads to the ion temperature, provided the ion mass is known. The height of the shoulders gives the electron to ion temperature ratio. Finally, in tristatic mode, the EISCAT radar gives access to the parallel and perpendicular electric field.

From the four main parameters measured by EISCAT (ion velocity, electron and ion temperatures, electron density), it is possible to deduce the meridional neutral wind (Lilensten et al., 1992). The idea is that, as seen from the radar, the ion velocity is the sum of the neutral velocity and the diffusion of the ions through the neutrals. The diffusion velocity depends on the temperatures and densities measured by EISCAT. We then solve the diffusion equation, and compare the meridional velocity measured by the interferometer with that deduced from the radar measurements (Fig. 1). Over the three winter campaigns, only six nights were available for such a comparison. For a typical average of $2 \mathrm{~h}$ on the radar results, the two winds agree quite well. However, for instantaneous time, one could see strong 

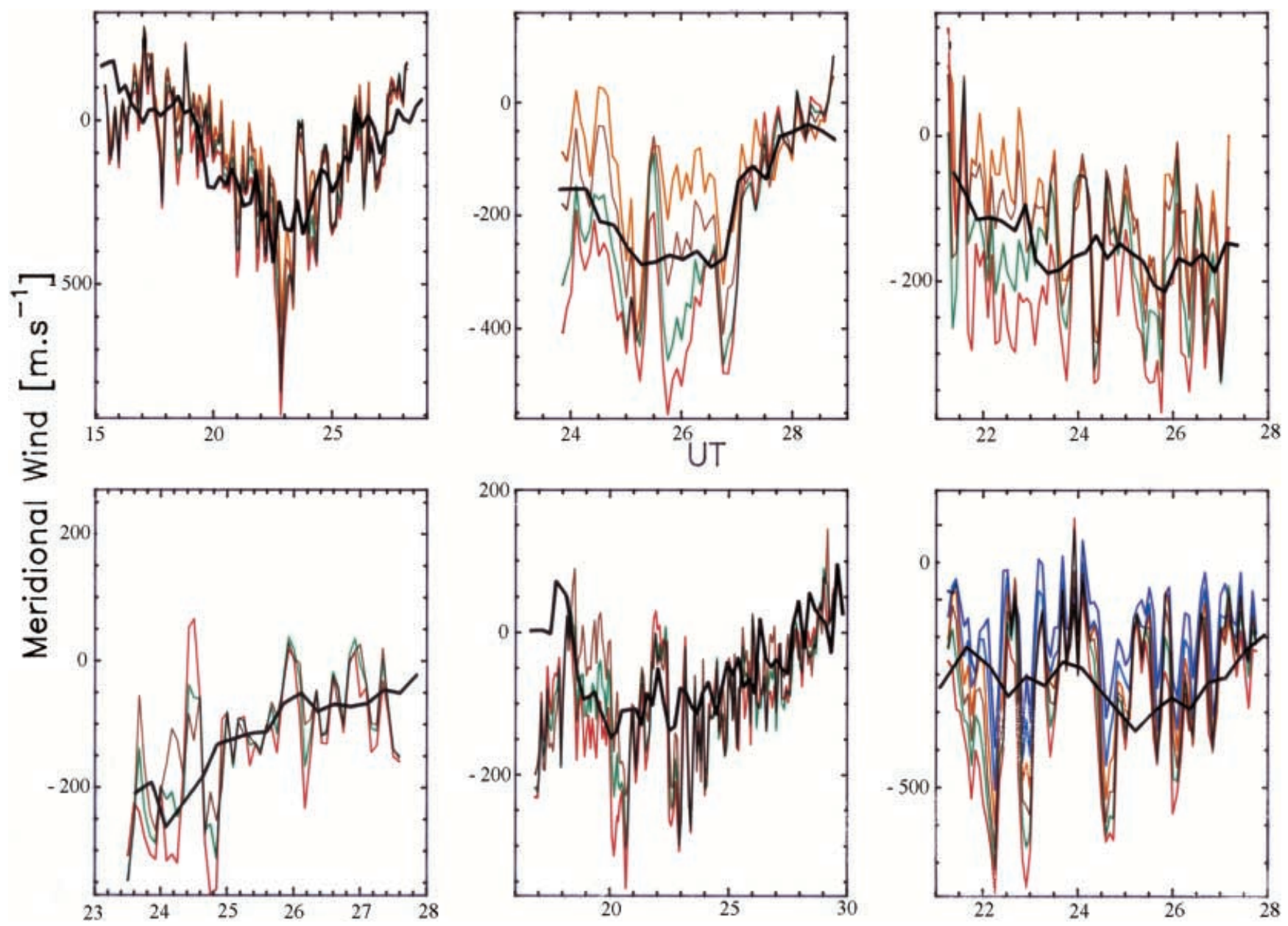

Fig. 1. Six nights of common EISCAT/MICADO coordinated observations (Lilensten et al., 1992). The interferometer wind is shown by a bold black line; the EISCAT derived wind in different colors. The values of the meridional wind is negative toward the south and positive to the north. The time is in UT, and a time larger than 24 means after midnight. Top left panel is the night of February 8 to 9, 1989. The altitudes are $212.5 \mathrm{~km}$ (orange), $234.5 \mathrm{~km}$ (brown), $256.5 \mathrm{~km}$ (green), $278.5 \mathrm{~km}$ (red). Top middle panel is the night of February 4 to 5, 1989. The altitudes are $168.5 \mathrm{~km}$ (orange), $190.5 \mathrm{~km}$ (brown), $212.5 \mathrm{~km}$ (green), $234.5 \mathrm{~km}$ (red). Top right panel is the night

oscillations of the radar-deduced wind around the interferometer measurement. Those oscillations occurred almost at any time. At that time though, it was not possible to deduce whether they were ionospheric or thermospheric movements. On the ionospheric side of the equation is the fact that the diffusion, equation is stationary. We tested this assumption, but adding non stationarity only introduced a decreasing exponential factor with very short (less than a second) and small effects. On the thermospheric side is the fact that the interferometer needs a full pointing cycle in order to make one meridional wind measurement. This means that it looks to the zenith, and then to different geographic positions (north, east, west and south). That takes about $15 \mathrm{~min}$, which is of the order of the oscillations. Moreover, there is a spatial averaging due to this cycle of about $300 \mathrm{~km}$ at the altitude of the red line emission (about $250 \mathrm{~km}$ height). of December 16 to 17,1989 . The altitudes are $234.5 \mathrm{~km}$ (orange), $256.5 \mathrm{~km}$ (brown), $278.5 \mathrm{~km}$ (green), $300.5 \mathrm{~km}$ (red). Bottom left panel is the night of January 28 to 29,1990 . The altitudes are $168.5 \mathrm{~km}$ (brown), $190.5 \mathrm{~km}$ (green), $212.5 \mathrm{~km}$ (red). Bottom middle panel is the night of December 20 to 21, 1990. The altitudes are $168.5 \mathrm{~km}$ (brown), $190.5 \mathrm{~km}$ (green), $212.5 \mathrm{~km}$ (red). Bottom right panel is the night of December 25, 1991. The altitudes are $168.5 \mathrm{~km}$ (purple), $190.5 \mathrm{~km}$ (blue), $212.5 \mathrm{~km}$ (orange), $234.5 \mathrm{~km}$ (brown), $256.5 \mathrm{~km}$ (green), $278.5 \mathrm{~km}($ red $)$

We decided to compare our measurements to the classical wind model by Hedin (1991). Taking different EISCAT experiments averaged over one to two hours, we could perform a close comparison, and identify some typical behavior of the high-latitude thermospheric wind (Lilensten and Lathuillère, 1995). However, without averaging, the oscillations were always present. The next step was to explore their coherency: if a time-frequency analysis could indicate a typical behavior, the wave could then be identified. We analyzed more than 20 experiments (Lilensten and Amblard, 1996). In some cases, we could clearly see classical gravity waves, but most of the time, no coherency was found: the oscillations could have any period between 5 to $30 \mathrm{~min}$.

A major step was performed when we could compare the EISCAT data to the WINDII measurements (Lathuillère et al., 1997).The Wind Imaging Interferometer (WINDII) on board the UARS satellite is described in 
detail by Sheperd et al. (1993). Its mission was to measure neutral winds, temperatures and emission rates in the altitude range 80 to $300 \mathrm{~km}$. The calibration work was performed on the wind measurements (Gault et al., 1996; Thuillier et al., 1996). The big advantage of WINDII is that the measurements are instantaneous and do not perform spatial averaging. We could compare 11 passes of the WINDII field-of-view near the radar facility. For the eight occasions when geomagnetic activity was low, the average difference in the meridional winds measured by the two methods was less than $10 \mathrm{~m} / \mathrm{s}$. The three passes corresponding to disturbed conditions showed poor agreement, as expected since the diffusion equation does not take the electric field into account. Moreover, the agreement between EISCAT and WINDII was better when unfiltered EISCAT winds were used, rather than the $2 \mathrm{~h}$ running mean used in the earlier work. This clearly suggested that the short-term oscillations seen by EISCAT are real oscillations of the neutral atmosphere. The present work is an attempt to explain this thermospheric pattern.

\section{The "tourbillons" (eddies)}

We first explored an electrodynamic interpretation. Electrons and ions are carried by the neutral gas, and on their way to the pole (equator), travel through an increasing (decreasing) magnetic field. This results in electromotive forces which slow down or accelerate the charged particles, but not the neutrals. From this, some waves could be generated.

However, the calculations show that the change in the magnetic field intensity is not sufficient to generate the observations. Anyway, the WINDII observations showed that the phenomena is a neutral one, not an ionospheric one. Therefore, this assumption had to be ruled out.

Our next step was to suppose that we are in fact dealing with eddies (or "tourbillons" in French). Since the radar is only able to see the north/south wind, we only observe the projection of the tourbillons on this axis, which results in the oscillations (Fig. 2). Those oscillations are not observed in by the optical observations simply because they are both too wide and too slow.

First of all, we must consider that in the typical time of these oscillations, there are many neutral/ion collisions. Namely, the product $v_{\text {in }} \cdot T$ is about 1000 , much bigger than 1. $v_{\text {in }}$ represents the ion-neutral collision (about $1 \mathrm{~s}^{-1}$ ) and $T$ the characteristic time of the oscillations (of the order of $1000 \mathrm{~s}$ ). Therefore, the dynamics of the neutrals is involved, rather than the dynamics of the ions. Then, we must check whether the atmosphere is turbulent enough to generate the tourbillons. This can be performed by estimation of the Reynolds number, defined as:

$R=\frac{U D}{v}$

where $U$ is the global velocity of the eddy, $D$ its diameter and $v$ is the kinematic viscosity. For our purpose, it is
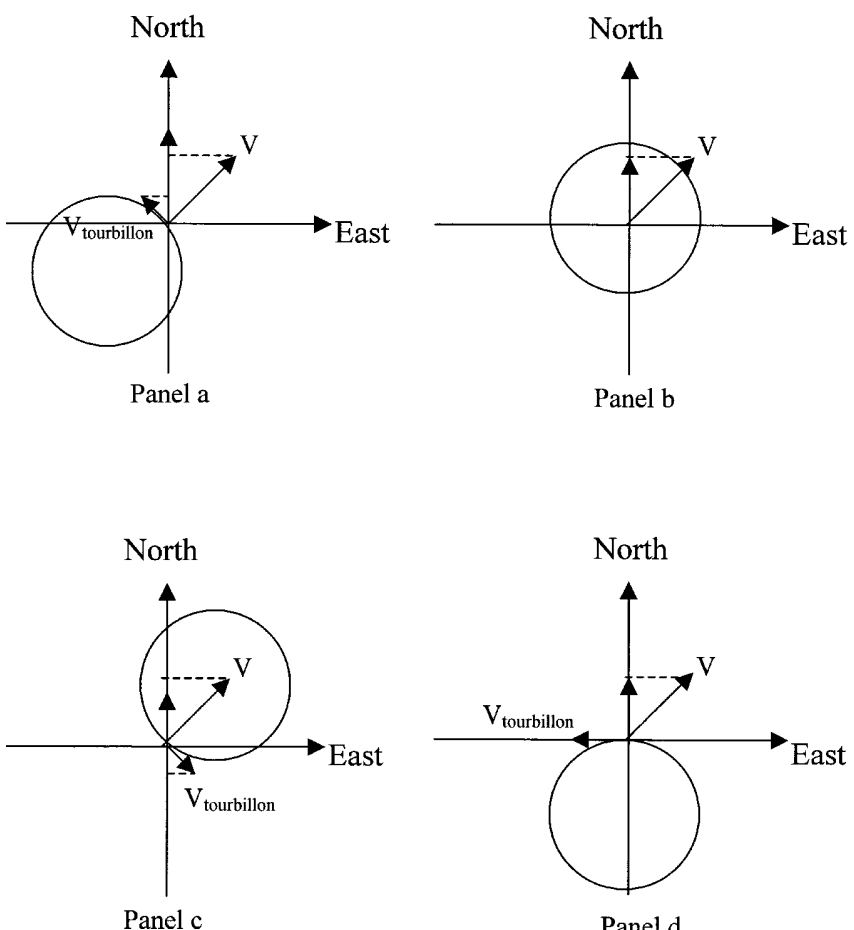

Fig. 2a-d. On this sketch, we plot the principle of the meridional wind projection. EISCAT is where two axes cross and observes along the magnetic field line perpendicularly to the sketch. The EISCAT deduced wind results from the sum of the global velocity $V$ and the eddy velocity $\left(V_{\text {tourbillon }}\right)$. In a the two are added to obtain a large meridional component. The meridional wind observed $\mathbf{b}$ corresponds to the single global displacement since then, the eddy velocity is zero above EISCAT. c Finally, the wind is lowered since the tourbillon velocity is subtracted from the global one. $\mathbf{d}$ The observed projection of the eddy velocity is also zero when the eddy velocity is perpendicular to the north-south axis

not necessary to determine precise values of the Reynolds number: we want to check the validity of our assumption.

The mean meridional wind $\left\langle V_{\text {merid }}\right\rangle$ constitutes a lower boundary for the value of the global velocity of the tourbillon. If the tourbillon goes fully north or south, then what is measured is this global velocity. If the tourbillon has an east-west component, what is measured on the north-south axis is smaller than its global velocity as seen from an observer at ground. The mean meridional wind $\left\langle V_{\text {merid }}\right\rangle$ is what is actually measured by the interferometer.

$D$ is the integral scale at which the energy is put in the system. It can be minimized by the diameter of the eddy, which may be determined from the period of the rotation $\tau$, and the rotation velocity. The rotation velocity is simply the difference between the mean meridional wind and, within one oscillation, the maximum of the meridional velocity. Then, we obtain:

$D \leq \frac{\left\{V_{\text {merid }}-\left\langle V_{\text {merid }}\right\rangle\right\} \tau}{\pi}$

The kinematic viscosity is the ratio between the dynamic viscosity $\mu\left[\mathrm{kg} \mathrm{m}^{-1} \mathrm{~s}^{-1}\right]$ and the density $\rho$. From Banks and Kockarts (1973), we have: 
$\mu(\mathrm{O})=3.9 \times 10^{-7} T^{0.69}$

$\mu\left(\mathrm{O}_{2}\right)=4.03 \times 10^{-7} T^{0.69}$

$\mu\left(\mathrm{N}_{2}\right)=3.43 \times 10^{-7} T^{0.69}$

For an atmosphere made of half atomic oxygen, and a quarter of each molecule, we have a dynamic viscosity of:

$\mu=3.8 \times 10^{-7} T^{0.69}$

$R \geq \frac{\left\langle V_{\text {merid }}\right\rangle \rho}{3.8 \times 10^{-7} T^{0.69}} \times \frac{\left\{V_{\text {merid }}-\left\langle V_{\text {merid }}\right\rangle\right\} \tau}{\pi}$

As an example, we can focus on the oscillation of 28 January, 1990, between 24 and 25 h (Fig. 3). It is neither the biggest nor the smallest. At that time, their is also a meridional wind, which is not known, and can only be estimated through a model. The FTH model (Fauliot et al., 1993) forecasts a zonal wind of more than $100 \mathrm{~m} \mathrm{~s}^{-1}$. Since we cannot be sure that it is the exact value of the zonal wind at that time, we will not take it into account. Would we consider it, we would still enhance the Reynolds number, and go in the sense of an increased turbulence. We will consider the values of the atmosphere at $168 \mathrm{~km}$, which is the lowest on this figure. The reason is that when one goes to very high altitudes (above typically $300 \mathrm{~km}$ ), the atmosphere density becomes so small that there is a great deal of doubt whether a tourbillon can originate. The data show that the oscillations are not as clear at $400 \mathrm{~km}$, where the

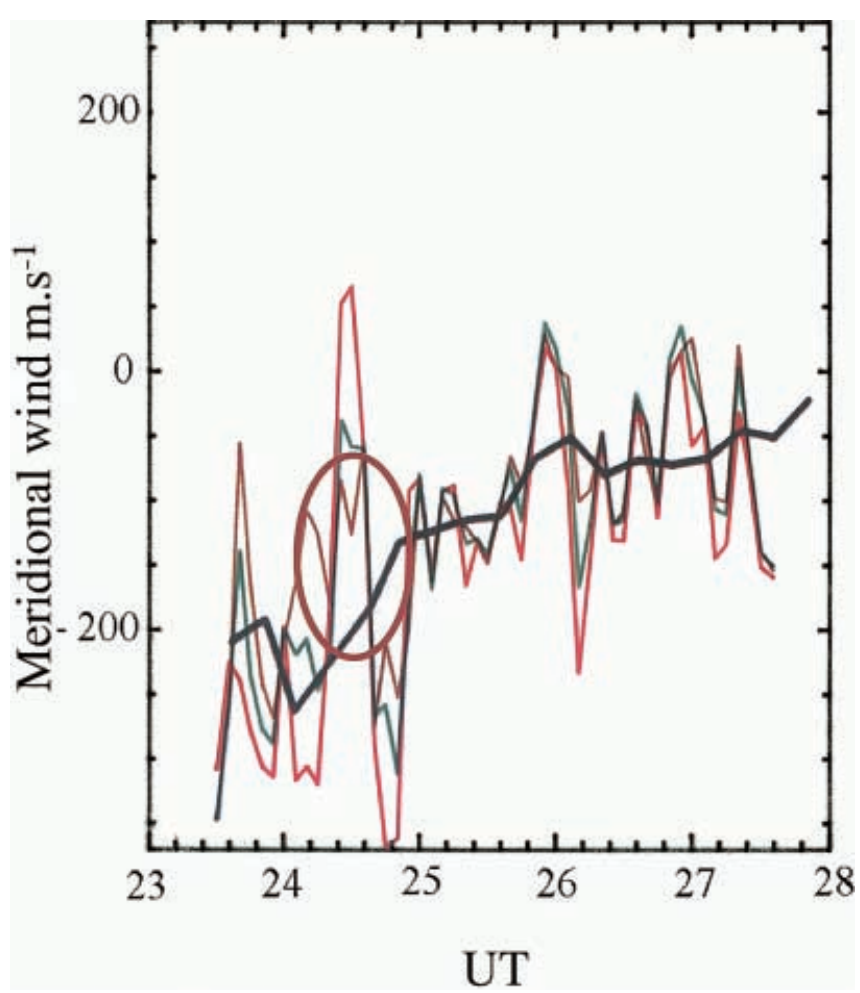

Fig. 3. This figure is an enlargement of the bottom left panel in Fig. 1, i.e., the night of January 28 to 29,1990 . The altitudes are $168.5 \mathrm{~km}$ (brown), $190.5 \mathrm{~km}$ (green), $212.5 \mathrm{~km}$ (red). The oscillation on which the computation is made in the text is encircled in brown atmosphere is collisionless. On the contrary, we think that the source of the eddies is at lower altitudes, probably where the gravity waves are created. From Fig. 1, we then take the following values:

$\left\langle V_{\text {merid }}\right\rangle \approx 200 \mathrm{~m} \mathrm{~s}^{-1}$

$V_{\text {merid }}-\left\langle V_{\text {merid }}\right\rangle \approx 200 \mathrm{~m} \mathrm{~s}^{-1}$

$\tau \approx 2900 \mathrm{~s}$

With these values, one obtains a diameter close to $200 \mathrm{~km}$. From the other oscillations seen in the EISCAT measurement, we have diameters varying from typically 50 to about $300 \mathrm{~km}$. From the MSIS neutral atmosphere model (Hedin, 1991), we determine:

$\mathrm{N} \approx 10^{16} \mathrm{~m}^{-3}$

$\mathrm{T} \approx 900 \mathrm{~K}$

$\mathrm{m} \approx 24 \times 1.661 \times 10^{-27} \mathrm{~kg}$

This gives a Reynolds number larger than 370. Following Frisch (1995), this corresponds to a non-fully developed turbulence, i.e. a number sufficient to create the eddies without breaking them. The eddies can be created for Reynolds numbers as low as 105 under the shape of Karman vortex streets. The turbulence is fully developed for numbers typically larger than 2000 (Lesieur, 1994). Then, the eddies can be broken in smaller scales.

One could object that the tourbillons occur even when the meridional wind is close to zero (example at 16 UT, on 4 February, 1989). However, at that time, the zonal wind has typical values of $300 \mathrm{~m} \mathrm{~s}^{-1}$ (Fauliot et al., 1993) for a local $\mathrm{K}$ index larger than 4 .

The case that we have taken is not the most favorable (nor the least favorable). For example, at the same time, one could compute the Reynolds number at the two other altitudes (190.5 and $212.5 \mathrm{~km})$. The dynamic viscosity is not much different because the neutral temperature does not vary much between these altitudes. The kinematic viscosity decreases because the density decreases, but at the same time, the difference between the meridional wind and its mean increases, so that the Reynolds number remains in the range of the developed turbulence. The values would be smaller on the 28 February, 1989, and larger on 16. The difficulty is that the tourbillons may be close to each other, and eventually turn in different directions, so that they are not easy to identify in each event.

Therefore, we think that the oscillations observed with the radar are due to the tourbillons. These features occur practically at any time. This suggests that it is very probably the normal way the wind propagates.

\section{Discussion and conclusion}

In fact, curls have already been observed many times through optical experiments. As early as 1981, a monograph shows nice curl structures in auroral arcs (Akasofu and Kan, 1981). The aurora changes in brightness, shape, color, dynamics, and location in 
response to changes in the state of the magnetosphere. This is exemplified by substorms, which cause an abrupt brightening of the most equatorward discrete arc in the midnight sector of the auroral oval and a disruption of the quiet-time nightside arc system (the "auroral breakup"). As more energy is released during the substorm expansion phase, in a series of pulse-like intensifications, the emissions in the midnight and pre-midnight sectors become very bright (a few hundred kilo-Rayleighs) and form a luminous bulge that expands into the polar cap. This "auroral bulge" is made up of a variety of highly dynamic forms, arc fragments, spirals, curls, pulsating patches, etc. At the end of the expansion phase, the aurora begins to dim and becomes less active; the nightside portion of the oval thins and returns to its quiet-time configuration and location. In these cases, the curls are the result of a magnetosphere/ionosphere relationship. Their scale is larger than the scale of the tourbillons that are referred to here (see e.g., the Dynamics Explorer gallery http://pluto.space.swri.edu/ IMAGE/glossary/aurora3.html).

Optical observations using high-resolution television cameras frequently show that auroral curls are associated with shear velocities in the apparent optical flow (Vogt et al., 1999). In this case, small-scale quasiperiodic distortions are subject to sudden decay rather than development into vortices. There, the auroral acceleration processes need to be taken into account. However, small scale tourbillons are observed even outside of bright auroras. Moreover, the visible curls can only be seen below typically $160 \mathrm{~km}$, which is the lower boundary of the red line emission.

The eddies are neutral wind features. The global electron/ion density remains globally unchanged, as well as the electron and ion temperatures. That is why these parameters do not exhibit the same oscillation patterns. It is not the same when one considers the electric field. This one is indeed the vector product of the ion velocity and the magnetic field. The fact that electric-field oscillations are seen in the EISCAT measurements is not a proof though, since both ion velocity and electric field come from the same measure, and independent measurements should be performed. However, the mobility of the electrons and ions is different, and a cross-tourbillon electric-field could then appear, adding to the perturbations of the global electric field.

In order to check our assumption, an experiment could be set up. It consists in the observation of the same geographical point by three different cross-calibrated interferometers, based on a large triangle, exactly such as made by the tristatic EISCAT radar (Fig. 4). A difference between the radar and the interferometers is that in the latter case, the observations are integrated over the field of view, so that they take into account the emissions from above and below the crossing point. However, the peak altitude of the red line emission is relatively narrow, and the emission decreases rapidly above and below this maximum (Witasse et al., 1999). With such an experiment, if our hypothesis is correct, the oscillations should show up on the meridional component as well as on the zonal one, with a $\pi / 2$ difference of phase. That

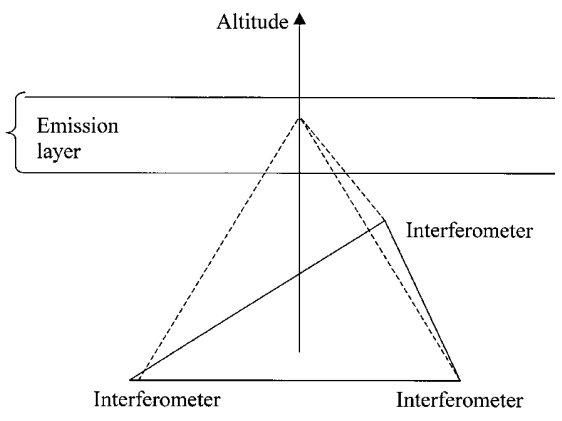

Fig. 4. Possible experiment to check the validity of the tourbillon hypothesis

could basically be made with only two interferometers, one observing the west-east axis, and the other the north-south one. The point in having a third device is that it is then possible to invert the vertical profile from tomographic methods (Solomon, 1987) and therefore avoid any ambiguity in the results.

Acknowledgements. We want to thank H. Gallee and O. Brasseur from the HMG laboratory for very pertinent discussions on turbulence. We also thank Alan Aylward for his encouraging support.

Topical Editor Mark Lester thanks M. Kasch for his help in evaluating this paper.

\section{References}

Akasofu, S. I., and J. R. Kan, Physics of auroral arc formation, Geophysical monographs, AGU Series, Washington, DC., 1981.

Banks, P. M., and G. Kockarts, Aeronomy, part $A$ and B, Academic Press, New York, 1973.

Fauliot, V., G. Thuillier, and M. Hersé, Observations of the Fregion horizontal and vertical winds in the auroral zone, Ann. Geophysicae, 11, 17-28, 1993.

Frisch, U., Turbulence, Cambridge University Press, ISBN 0521 $451030,1995$.

Gault, W. A., et al., validation of $\mathrm{O}(1 \mathrm{~S})$ wind measurements by WINDII: the wind imaging interferometer on UARS, J. Geophys. Res., 101, 405-430, 1996.

Hedin, A. E., Extension of the MSIS thermosphere into the middle and lower atmosphere, J. Geophys. Res., 96, 1159-1172, 1991.

Lathuillere, C., J. Lilensten, W. Gault, and G. Thuillier, The meridional wind in the auroral thermosphere: results from EISCAT and WINDII-O1D coordinated measurements, J. Geophys. Res., 102, 4487-4492, 1997.

Lesieur, M., La turbulence, Presses Universitaires de Grenoble, collection Grenoble Sciences, ISBN 270610588 7, 1994.

Lilensten, J., and C. Lathuillere, The meridional thermospheric neutral wind measured by the EISCAT radar, J. Geomagn. Geoelectr, 47, 911-920, 1995.

Lilensten, J., and P. O. Amblard, The use of time-frequency tools of signal processing for EISCAT data analysis, Ann. geophysicae, 14, 1513-1525, 1996.

Lilensten, J., G. Thuillier, C. Lathuillere, W. Kofman, V. Fauliot, and M. Hersé, EISCAT-MICADO coordinated measurements of meridional wind, Ann. Geophysicae, 10, 603-618, 1992.

Rishbeth, H., and A. P. Van Eyken, EISCAT: early history and the first ten years of operation, J. Atmos. Terr. Phys., 55, 525-542, 1993.

Sheperd, G. G., et al., WINDII: the wind imaging interferometer on the Upper Atmosphere Research Satellite, J. Geophys. Res., 98, 1303-1306, 1993. 
Solomon, S. C., Tomographic inversion of auroral emissions, technical report DRDA Project 020927, NASA, Washington D.C., 1987.

Thuillier, G., and M. Hersé, Measurements of wind in the upper atmosphere: first results of the MICADO instrument, Progr. Atmos. Phys., Kluwer, Dortrecht, The Netherlands, 61-73, 1988.

Thuillier, G., V. Fauliot, M. Hersé, L. Bourg, and G. G. Sheperd, The MICADO wind measurements from Observatoire de Haute
Provence for the validation of the WINDII green line data, J. Geophys. Res., 101, 431-440, 1996.

Vogt, J., H. U. Frey, G. Haerendel, H. Hofner, and J. L. Semeter, Shear velocity profiles associated with auroral curls, J. Geophys. Res., 104, 17 277-17 288, 1999.

Witasse, O., J. Lilensten, C. Lathuillere, and P. L. Blelly, Modeling the OI 630.0 and $557.7 \mathrm{~nm}$ thermospheric dayglow during EISCAT-WINDII coordinated measurements, J. Geophys. Res., 104, 24 639-24 655, 1999.

Ann. Geophysicae 18, 1656 (2001) C EGS - Springer-Verlag 2001

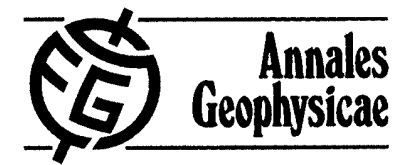

\section{Erratum}

Published in Annales Geophysicae Volume 18, number 9, on page 1009, 2000

In the paper McCrea et al., Vol. 18, number 9, p. 1009, entitled: "ESR and EISCAT observations of the response of the cusp and cleft to IMF orientation changes", one of the co-authors Dr. A. Thorolfsson is erroneously called A. Thorolfssen. We apologize for this mistake. The correct authors list is:
I. W. McCrea, M. Lockwood, J. Moen, F. Pitout, P. Eglitis, A. D. Aylward, J.-C. Cerisier, A. Thorolfsson, S. E. Milan. 